\title{
Atomistic migration mechanisms of atomically flat, stepped, and kinked grain boundaries
}

\author{
R. Hadian, ${ }^{1, *}$ B. Grabowski, ${ }^{1}$ C. P. Race, ${ }^{2}$ and J. Neugebauer ${ }^{1}$ \\ ${ }^{1}$ Max-Planck-Institut für Eisenforschung GmbH, Max-Planck-Str. 1, 40237 Düsseldorf, Germany \\ ${ }^{2}$ School of Materials, University of Manchester, Manchester M13 9PL, United Kingdom
}

(Received 18 March 2016; revised manuscript received 13 September 2016; published 13 October 2016)

\begin{abstract}
We studied the migration behavior of mixed tilt and twist grain boundaries in the vicinity of a symmetric tilt $\langle 111\rangle \Sigma 7$ grain boundary in aluminum. We show that these grain boundaries fall into two main categories of stepped and kinked grain boundaries around the atomically flat symmetric tilt boundary. Using these structures together with size converged molecular dynamics simulations and investigating snapshots of the boundaries during migration, we obtain an intuitive and quantitative description of the kinetic and atomistic mechanisms of the migration of general mixed grain boundaries. This description is closely related to well-known concepts in surface growth such as step and kink-flow mechanisms and allows us to derive analytical kinetic models that explain the dependence of the migration barrier on the driving force. Using this insight we are able to extract energy barrier data for the experimentally relevant case of vanishing driving forces that are not accessible from direct molecular dynamics simulations and to classify arbitrary boundaries based on their mesoscopic structures.
\end{abstract}

DOI: 10.1103/PhysRevB.94.165413

\section{MOTIVATION}

The migration of grain boundaries plays a pivotal role in the evolution of materials microstructures, which strongly impacts the mechanical, chemical, or electronic response of a material. Thus, for designing optimized materials with tailored microstructures, a detailed understanding of grain boundary kinetics and of the underlying atomistic mechanisms is crucial. Identifying these fundamental mechanisms and understanding their impact on grain boundary migration has been a key topic both from an experimental as well as a theoretical perspective for several decades.

In 1948 Mott [1] proposed that the migration is a thermally activated process. In his model for migration, the energy barrier that the boundary must overcome is related to an island nucleation mechanism where atoms melt on one side of the boundary and solidify on the other crystalline side. In 1969 Gleiter portrayed a general grain boundary as a 3D stepped structure by interpreting diffraction contrast microscopy images $[2,3]$. He proposed that general nonsymmetric grain boundaries are not atomically flat but are rather comprised of atomic steps and kinks, similar to the well-known structural elements on surfaces. He also pointed out that the boundary moves via the emission and absorption of atoms at step kinks.

The picture of both Mott [1] and Gleiter [2,3] that grain boundaries consist of an intermediate layer changed with the advent of high-resolution electron microscopy which showed that the interface region is rather sharp [4]. In the late 1970s several electron microscopic studies analyzed grain boundary migration in terms of the motion of secondary grain boundary dislocations [5-7]. However a more recent in situ electron microscopy work by Babcock and Balluffi [8] in 1989 showed the contribution from the dislocation motion to be negligible. In their study [8] of curvature-driven grain boundary migration in near $\Sigma 5$ boundaries the authors proposed that migration mainly takes place by atomic shuffling around pure steps in the boundary.

\footnotetext{
*Corresponding author: r.hadian@mpie.de
}

In the last 20 years theoretical simulations have become increasingly useful to supplement and interpret the experimental studies. Due to computational constraints the first studies employed Monte Carlo simulations in the framework of a twodimensional Ising model which allowed the study of motion of model boundaries. Despite being rather idealized these models highlighted the importance of island and kink nucleation and propagation on the motion of grain boundaries [9-11]. As more powerful computers emerged, the theoretical efforts were extended to three-dimensional molecular dynamics (MD) simulations providing a more realistic description of boundary migration. These tools led to the identification of cooperative motion of atom clusters in the migration of high angle grain boundaries [12,13], confirming the experimental results of Babcock and Balluffi [8].

Generally, the focus of the MD studies was on cooperative mechanisms rather than on mesoscopic features and mechanisms such as kinks or steps that had been highlighted in the earlier experimental and theoretical papers. One reason may be that the computational constraints have limited these calculations to rather small supercells. As has been discussed in a recent study [14] small supercell sizes may inhibit the formation of the correct dynamical mesostructure with the consequence that unphysical mechanisms prevail and determine the rate of boundary migration, particularly when approaching the comparatively small driving forces characteristic of experimental cases. Another typical limitation of MD simulations has been the restriction to symmetric tilt boundaries. Recent studies showed that such symmetric grain boundaries are only partly representative of experimentally observed grain boundaries in polycrystalline materials [15-17]. The migration of grain boundaries with general, nonsymmetric planes has not been intensively studied, and only very recent theoretical works have established the significance of grain boundary plane orientation in migration [18-20].

The aim of the present study is to employ MD simulations to study the atomistic mechanisms involved in the migration of grain boundaries with nonsymmetric plane orientations in the vicinity of a symmetric tilt one. We will show that these nonsymmetric grain boundaries relax into nanofaceted 
structures [21-24]. As a consequence only two distinct categories of stepped structures result: straight steps without any kinks or steps with periodic arrays of kinks. For both categories we investigate the velocity of these boundaries along with the atomically flat symmetric tilt grain boundary and probe the time evolution of their mesoscopic features. We thereby combine the two major research directions in modeling grain boundary migration by reintroducing and studying the concepts of mesoscopic migration mechanisms in realistic grain boundaries. Based on these results we show qualitatively different migration morphologies of the three boundary types (flat, stepped, and kinked) and link them to well-known growth modes in surface science (see Sec. IV A). Using this insight we are able to derive the asymptotic behavior of the grain boundaries (see Sec. IV B), which allows us to connect the much faster time scale of the MD simulations with the experimental time scale.

\section{SIMULATION METHODS}

To study the above mentioned grain boundary types we explored mixed tilt and twist grain boundaries in the vicinity of the symmetric $\Sigma 7$ tilt boundary in Al. Al was chosen since, due to its high purity and high crystalline quality, it is a preferred system for experimental grain boundary studies [25] and because well established empirical potentials are available [26]. The specific grain boundary system, $\Sigma 7$ tilt, has been extensively investigated in previous theoretical studies $[18,27,28]$. All calculations were performed using the LAMMPS code [29]. The interatomic forces were modeled by the aluminum embedded-atom-method (EAM) potential developed by Zope and Mishin [26]. This potential has been fitted to $\mathrm{Al}$ properties from both experiment and $a b$ initio calculations. Specifically, the calculated symmetrical twin boundary energy using this potential agrees well with the experimental value [26].

The grain boundary structures were constructed within a coincident site lattice (CSL) approach. They were obtained by introducing a small inclination to the symmetric boundary plane as indicated in Figs. 1(a)-1(c), with the inclination axis chosen to lie inside the boundary plane. Aside from the [111] direction (i.e., the $\Sigma 7$ tilt axis), rotation around any other inclination axis gives rise to a mixed tilt and twist grain boundary. Periodic boundary conditions were employed in all three dimensions due to which an image grain boundary is introduced along the boundary normal in the supercell. After running extensive convergence tests we chose a separation distance of at least $20 \mathrm{~nm}$ between the boundary and its periodic images to prevent any artificial interactions between them. The periodic boundary conditions can also introduce artificial correlations within the grain boundary plane. As shown recently, these artificial correlations can alter the migration mechanisms if the lateral supercell dimensions are too small [14]. Extensive benchmarks showed that this is less of a problem for our nonsymmetric grain boundary structures where the small misorientation angles from the symmetric

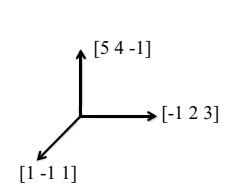

(a) Symmetric
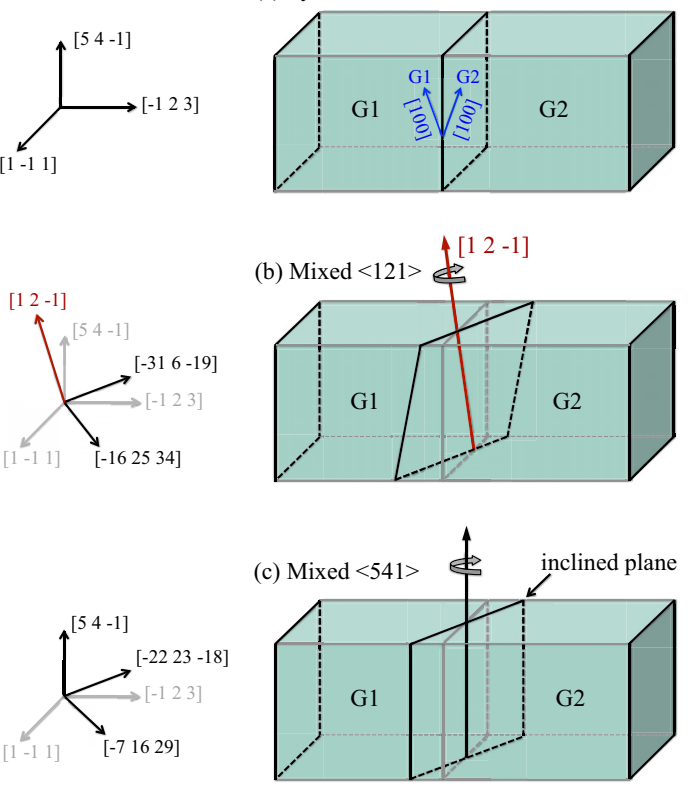
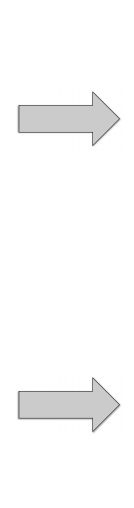

(d) Flat
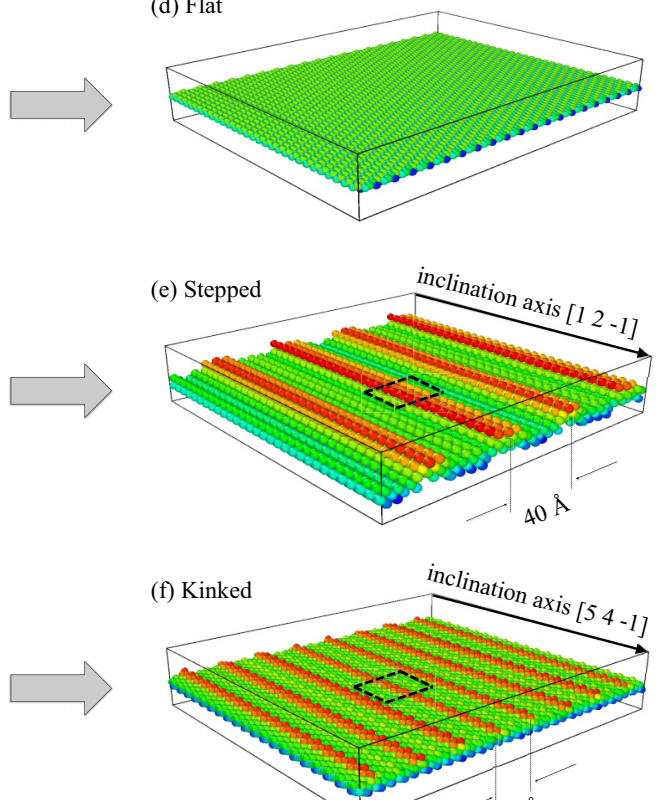

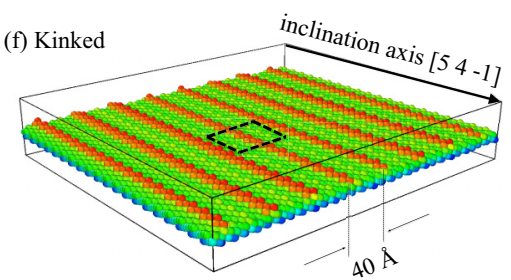

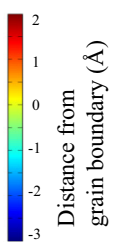

(g) Zoom Stepped

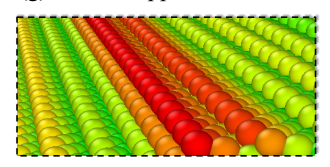

(h) Zoom Kinked

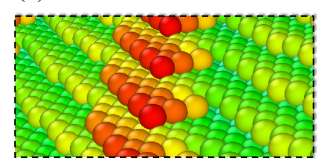

FIG. 1. Schematics of the investigated grain boundaries [(a) to (c)] and their static structures after $T=0 \mathrm{~K}$ relaxation [(d) to (h)]. (a) Pure symmetric $\Sigma 7$ tilt boundary with a misorientation angle of $38.21^{\circ}$ around the $\left[\begin{array}{lll}1 & 1 & 1\end{array}\right]$ axis, relaxing to an atomically flat structure as displayed in (d). (b) Mixed boundary obtained by introducing a small rotation angle around the [12 1)] direction in the grain boundary plane, relaxing to a structure with straight steps as shown in (e) and (g). (c) Mixed boundary obtained by introducing a small inclination angle to the symmetric boundary, around an arbitrary low symmetry rotation axis in the grain boundary plane as indicated by the black arrow. As a specific representation the [54 1 ] rotation axis has been chosen. This boundary relaxes to a structure with kinked steps as shown in (f) and (h). In (d) through (h) perfect bulk atoms have been removed for clarity, and the color indicates the position of the grain boundary atoms along the boundary plane normal as indicated by the color bar. 
boundary result in large lateral dimensions of the CSL unit cells. Nevertheless, we ensured that our grain boundary planes are size converged. For the structures and driving forces considered here we have therefore used areas of at least $20 \mathrm{~nm} \times 20 \mathrm{~nm}$ resulting in supercells of at least 500000 atoms.

In general, the as-constructed grain boundaries could not be directly utilized for migration simulations since they do not correspond to the thermodynamic equilibrium structure. To start the migration studies from an equilibrated grain boundary structure we have utilized a two step procedure: In the first step, the grain boundaries were subjected to a $T=0 \mathrm{~K}$ relaxation using a conjugate gradient approach. To detect the true energy minimum of the grain boundary and not only the nearest local minimum, we have explicitly investigated the microscopic degrees of freedom of each grain boundary [30]. To this end, we have constructed various initial configurations for the relaxation $(\approx 100$ per boundary) by applying rigid body translations of magnitudes smaller than the CSL repeat distance on the grain boundary plane (specifically, the in-plane displacement-shift-complete vector). In a second step, the lowest-energy grain boundary structures were then subjected to an annealing procedure using molecular dynamics simulations. In particular, the temperature of the system was first ramped up within a time frame of $50 \mathrm{ps}$ from $T=0 \mathrm{~K}$ to the target temperature for the migration simulations, and then the grain boundary was annealed for about $500 \mathrm{ps}$ at that temperature.

Migration simulations were then performed across a range of temperatures $(400-750 \mathrm{~K})$ and driving forces $(0.5-12 \mathrm{meV}$ ) for a few nanoseconds. To trigger grain boundary migration, we used a synthetic driving force that applies a potential energy bias to one of the grains determined by a misorientation-based order parameter $[18,28]$. This potential bias exerts forces of small magnitude (as compared to forces due to thermal fluctuations) on atoms in the vicinity of the grain boundary, which statistically favor the growth of one grain at the expense of the other. During the application of the driving force, a thermostat kept the temperature constant. It has been shown [31] that in the limit of small driving forces the synthetic force induces the same mobilities as an elastic strain driving force.

All molecular dynamics simulations were performed within an NPT ensemble. The Nosé-Hoover thermostat $[32,33]$ and the Parrinello-Rahman barostat [34] were used for controlling temperature and pressure, respectively. The time step for integrating the equations of motion was $1 \mathrm{fs}$.

To detect the grain boundary position, atomic coordinates were recorded every $100 \mathrm{fs}$ during the migration simulations. For each snapshot, a Gaussian broadening was applied to each atomic position to produce a smooth distribution $F\left(x^{\perp}\right)$ :

$$
F\left(x^{\perp}\right)=\sum_{i} w_{i}^{\text {ord }} \exp \left[-\frac{\left(x^{\perp}-x_{i}^{\perp}\right)^{2}}{\sigma^{2}}\right] .
$$

Here, $x^{\perp}$ is the position normal to the grain boundary plane, the index $i$ runs over all atoms, $x_{i}^{\perp}$ is the coordinate of the $i$ th atom normal to the grain boundary, $w_{i}^{\text {ord }}$ the corresponding weight given by the centrosymmetry order parameter (which equals zero for perfect fcc), and $\sigma$ a smoothing parameter. A value of $\sigma=0.05 \AA$ provided a smooth distribution $F\left(x^{\perp}\right)$ with a well defined maximum at the boundary where the centrosymmetry

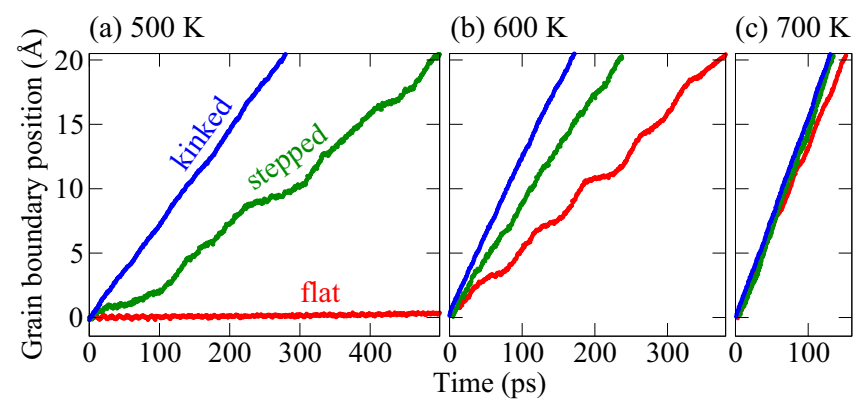

FIG. 2. Position versus time dependence of the three representative grain boundaries at (a) $500 \mathrm{~K}$, (b) $600 \mathrm{~K}$, and (c) $700 \mathrm{~K}$ subjected to a driving force of $5 \mathrm{meV}$ per atom. At $700 \mathrm{~K}$ all three boundaries have similar kinetics and the curves overlap.

parameter becomes the largest. Using the above equation we extracted the grain boundary position $X_{t}$ at time $t$ according to

$$
X_{t}=\left\{x^{\perp} \mid \max \left[F\left(x^{\perp}\right)\right]\right\} .
$$

Calculating $X_{t}$ as a function of simulation time provides an accurate dependence of grain boundary position versus time (cf. Fig. 2). The boundary velocity was then determined by means of linear regression.

To allow for a detailed investigation of the evolution of the mesoscopic structure, snapshots were recorded during the migration calculations. Each snapshot was subjected to a conjugate gradient minimization to remove thermal disorder. The resulting atomic configurations were analyzed using the visualization software Ovito [35].

\section{RESULTS}

\section{A. Flat, stepped, and kinked grain boundary structures}

Various mixed grain boundaries were constructed and relaxed at $T=0 \mathrm{~K}$ according to the procedure described in Sec. II. As opposed to the atomically-flat symmetric tilt grain boundary depicted in Fig. 1(d), the mixed grain boundaries form nanofacets/steps along the direction of the inclination axis [red features in Figs. 1(e) and 1(f)]. After an extensive search in the mixed grain boundaries around the symmetric tilt orientation, we found only two distinct categories of stepped structures, based on their structure and geometry. One category [Fig. 1(e)] is characterized by steps that lie along a high symmetry direction in the bicrystal, such as [1 $21 \overline{1}]$ in fcc and remain straight during minimization as shown in Fig. 1(g). In the other category [Fig. 1(f)] steps are lying along a low symmetry, rough direction such as [5 $4 \overline{1}]$ and are subfaceted (kinked) along high symmetry directions as shown in Fig. 1(h). In this case subfacets develop along the [12 $1 \overline{1}]$ and [1 10$]$ directions. In the following we focus on the two representative mixed grain boundaries in comparison with the symmetric tilt one. Henceforth, we refer to the boundaries that are symmetric tilt, mixed with straight steps and mixed with kinked steps as flat, stepped, and kinked for brevity.

The stepped and kinked boundaries consist of terrace planes of the symmetric plane orientation $\left\{\begin{array}{lll}1 & 2 & 3\end{array}\right\}$ (green areas) and steps of three atomic layers $(\approx 3 \AA$; red areas $)$. The steps 
are oriented along the direction of the inclination axis. The spacing between the nanofacets, i.e., their density, depends on the magnitude of the inclination angle. Similar nanofaceted structures have been previously observed in asymmetric tilt boundaries in the proximity of a symmetric boundary [21,22] as well. The stepped and kinked boundaries shown in Fig. 1 correspond to an inclination angle of about $6^{\circ}$ leading to a nanofacet spacing of about $40 \AA$. With increasing inclination angle, the spacing decreases such that at about $9^{\circ}$ such nanofaceted structures cannot be resolved anymore. Faceting will then happen on a low energy/high symmetry plane other than $\left\{\begin{array}{lll}1 & 2 & 3\end{array}\right\}$.

It has been discussed [30] that grain boundary steps may contain a dislocation component. A recently developed extraction method [36] identifies defects by mapping the atoms from the nanofaceted grain boundary structures to a reference structure. Using this method and the ideal symmetric tilt configuration as reference, we have investigated whether the steps/nanofacets of this study have dislocation character. Our results show that the mixed grain boundaries discussed above exhibit no dislocation character.

In some nonsymmetric grain boundaries in $\mathrm{Al}$, facet coarsening has been observed after annealing for several nanoseconds [37]. We have therefore performed long-time annealing simulations to test whether our mixed boundaries are stable with respect to facet coarsening. The results show that even after 2 ns of annealing the facet lengths remain unchanged except for thermally induced fluctuations. We have also monitored the grain boundary energy as a function of annealing time. Corresponding changes are below $1 \mathrm{~mJ} / \mathrm{m}^{2}$ indicating that the original structure is indeed stable.

\section{B. Migration kinetics}

Using the equilibrated grain boundary structures we computed the migration kinetics as a function of temperature. Figure 2 shows the position versus time dependence of the representative grain boundaries for three relevant temperatures at $5 \mathrm{meV} /$ atom. Three qualitatively different modes of migration can be observed: (i) immobile, (ii) stop-and-go, and (iii) continuous. Table I gives an additional overview of the migration modes and their correspondence to the grain boundary type and temperature.

The atomically-flat symmetric tilt boundary shows all three modes at the temperatures investigated. At low temperatures [500 K; Fig. 2(a)] this boundary is indeed immobile. At intermediate temperatures [600 K; Fig. 2(b)] a stop-and-go mode appears. A stop-and-go migration mode with increasing temperature is a characteristic of an activation/nucleation-

TABLE I. Migration modes as observed for the grain boundaries and temperatures in Fig. 2. "Immobile" refers to grain boundaries not moving within the accessible simulation time frame.

\begin{tabular}{|c|c|c|c|}
\hline & $500 \mathrm{~K}$ & $600 \mathrm{~K}$ & $700 \mathrm{~K}$ \\
\hline Flat & immobile & stop-and-go & continuous \\
\hline Stepped & stop-and-go & continuous & continuous \\
\hline Kinked & continuous & continuous & continuous \\
\hline
\end{tabular}

based migration mechanism: The boundary is stationary until the nucleation event occurs and moves until the next nucleation event is required. As nucleation is a stochastic process the stop periods in Fig. 2(b) are irregular. It was shown in Ref. [14] that the nucleation mechanism for a flat boundary is the formation of islands of atoms with the orientation of the growing grain. At higher temperatures [700 K; Fig. 2(c)] the flat boundary is above its roughening temperature, i.e., islands form spontaneously and the migration is no longer limited by island nucleation. It thus migrates in a continuous linear fashion.

The stop-and-go mode at $500 \mathrm{~K}$ observed for the stepped boundary implies that its migration is controlled by a nucleation mechanism [Fig. 2(a)]. This nucleation mechanism is different from the one active for the flat boundary: The stepped boundary is mobile at the lower temperature of $500 \mathrm{~K}$ which implies a lower energy barrier for the nucleation mechanism. The stop-and-go mode for this boundary appears to be less well defined than the one for the flat boundary. A stop-and-go mode of motion has also been observed in the literature of shear coupled motion [38]. Compared to the flat and stepped interfaces, the kinked boundary has a distinctly different migration behavior. The migration mode is continuous at all the temperatures shown, and it remains continuous even down to $400 \mathrm{~K}$ and at all driving forces. The absence of a stop-and-go migration mode is a clear indication that the migration of the kinked boundary is not controlled by nucleation but rather by an activation/nucleation-free mechanism.

These observations are a first indication of the qualitatively different migration behavior of the three grain boundaries. To gain further insight into the migration kinetics and identify the underlying mechanisms we performed an extensive study at various temperatures and driving forces. Figure 3 shows the corresponding results in an Arrhenius representation, i.e., logarithm of the velocity versus inverse temperature.

The flat boundary [Fig. 3(a)] shows a strongly nonArrhenius (nonlinear) relationship with temperature at all studied driving forces. The mesoscale island nucleation model proposed in Ref. [14] accurately reproduces the temperature dependence of the velocity below the roughening temperature using only two fitting parameters [see the dashed lines in Fig. 3(a)]. The dynamical roughening temperature (dotted line bounding the gray triangle) can be seen to increase with decreasing the driving force. This behavior has also been reported in Ref. [31].

For the stepped and kinked boundaries we find likewise a roughening transition temperature [black dotted lines in Figs. 3(b) and 3(c)] at which the temperature dependence of the velocity changes. While the transition temperatures of all three boundaries are similar, the Arrhenius dependence below this temperature shows a qualitatively different dependence for the stepped and kinked boundaries: In clear contrast to the quadratic dependence found for the flat boundary, the stepped and kinked boundaries show a linear behavior at all driving forces, except for some numerical scatter at low velocities due to the limited simulation times. This linear Arrhenius behavior for the migration of the mixed boundaries resembles the reported experimental migration kinetics for planar grain boundaries [25] and was also observed in our preliminary simulations of a nonplanar boundary [24]. 
(a) Flat

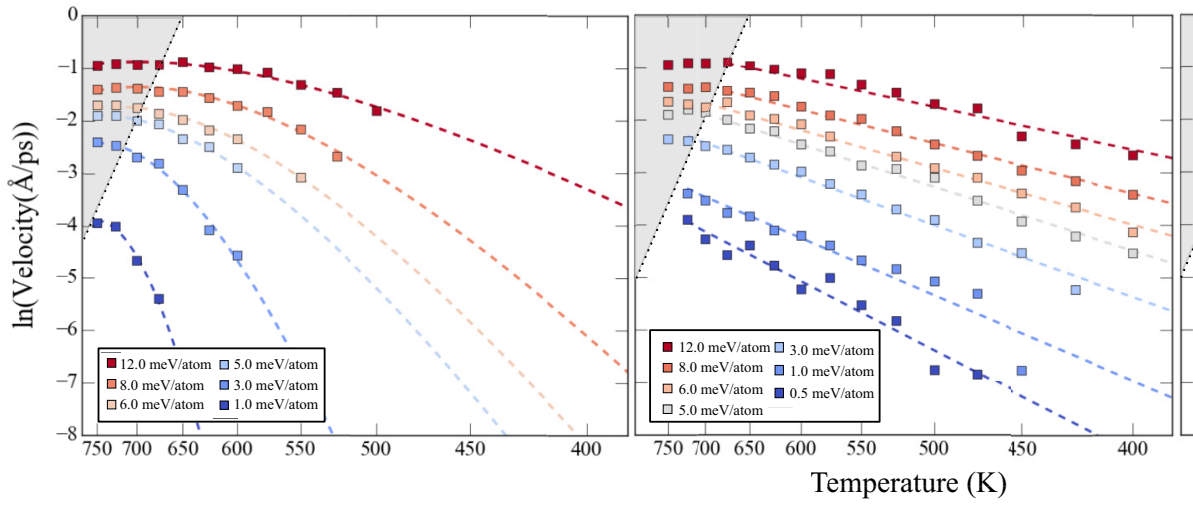

(c) Kinked

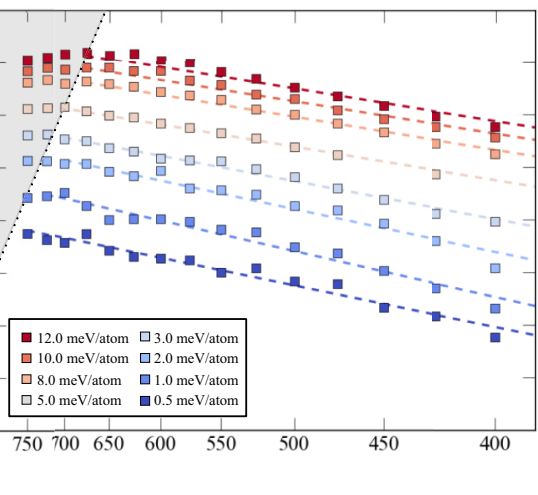

FIG. 3. Arrhenius representation of the grain boundary velocity as a function of inverse temperature at various driving forces for (a) flat, (b) stepped, and (c) kinked boundaries. The squares indicate the calculated data and the dashed lines are fits, quadratic for the flat boundary according to the island nucleation model [14] and linear for the stepped and kinked boundaries. The dotted black line indicates the roughening transition temperature of the grain boundaries, and the gray area marks the region above the transition temperature.

The linear Arrhenius dependence of the stepped boundary provides an additional argument that the nucleation mechanism of this boundary is markedly different from the island nucleation mechanism found for the flat boundary. The kinked grain boundary also shows a linear dependence in the Arrhenius representation as the stepped boundary. However, there is an important difference between the kinetics of the two boundaries: The slope of the fitted lines for the stepped boundary becomes steeper with decreasing driving force [Fig. 3(b)], whereas for the kinked boundary the slopes remain roughly the same [Fig. 3(c)]. The slopes represent an averaged migration energy barrier, and this difference in behavior indicates distinctly different migration mechanisms for the two mixed grain boundaries.

Figure 4 summarizes the extracted energy barriers for all three grain boundaries as a function of driving force. Figure 4(a) reveals a dramatic difference between the energy barriers for the flat boundary and the barriers for the stepped/kinked boundaries. As pointed out in Ref. [14] the divergent behavior of the energy barrier for the flat boundary at vanishing driving forces implies that this boundary becomes immobile at experimentally accessible driving forces $(<1$ $\mathrm{MPa}=0.1 \mathrm{meV} /$ atom from Ref. [25]). In contrast for both stepped and kinked boundaries we find well defined limits towards zero driving forces, implying that they are indeed mobile at experimental driving force limits.

\section{Atomistic mechanisms of migration}

Figures 5(a)-5(c) display representative migration snapshots for the three grain boundaries. Only grain boundary atoms are shown for clarity, and the color code is based on the distance to the grain boundary plane. On the flat boundary plane multiple islands are clearly visible [red areas in Fig. 5(a)] consistent with the kinetic behavior of this boundary discussed in the previous section. The snapshots in Figs. 5(b) and 5(c) for the stepped and kinked boundaries show instead a qualitatively different appearance.

For the stepped boundary with the steps along the high symmetry direction $\left[\begin{array}{lll}1 & 2 & 1\end{array}\right]$, steps remain straight across the grain boundary plane and can propagate only through nucleation and propagation of double kinks. The magnified region in Fig. 5(e) reveals a stable double kink nucleus on the triatomic steps (described in Sec. III A) along the [1 2 1 ] direction. An analysis of all our simulation runs for the stepped boundary shows that these double kinks frequently nucleate. Some of them are short-lived and quickly disappear (i.e., they are below their critical size), but many grow and coalesce. On the displayed stepped boundary [Fig. 5(b)] there exist four steps that advance by the nucleation, growth, and coalescence of double kinks.

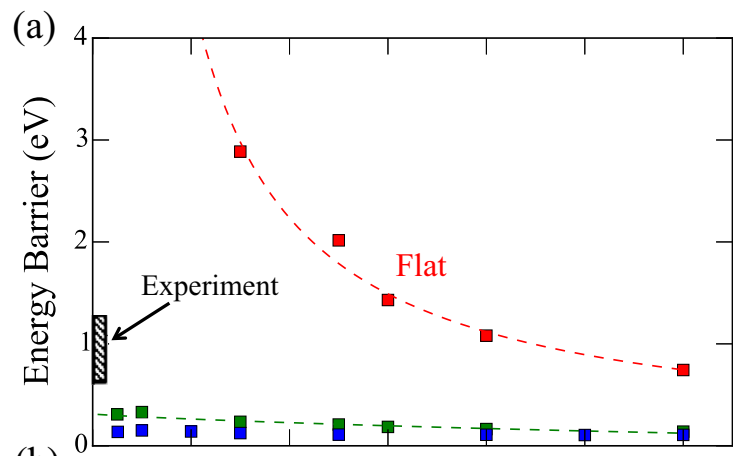

(b)

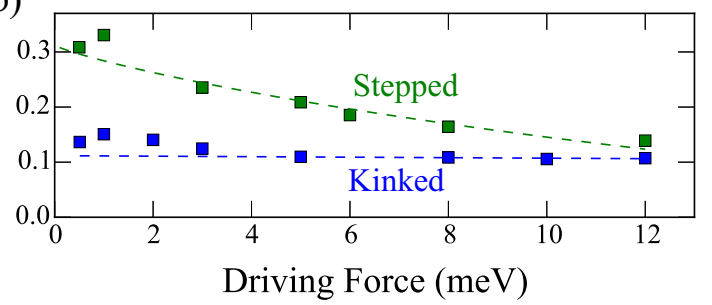

FIG. 4. Energy barriers extracted from the Arrhenius representation of Fig. 3 shown at (a) a small energy scale to capture all grain boundaries and (b) a larger scale to emphasize the dependence for the stepped and kinked boundaries. For the flat boundary, the fitted curve (red dashed line) was obtained using the island nucleation model [14]. The fit for the stepped boundary (green dashed line) is based on the model developed in Sec. IV A, and for the kinked boundary the fit is a constant (blue dashed line). The range of experimentally observed energy barriers [25] is indicated by the black bar at low driving forces. 


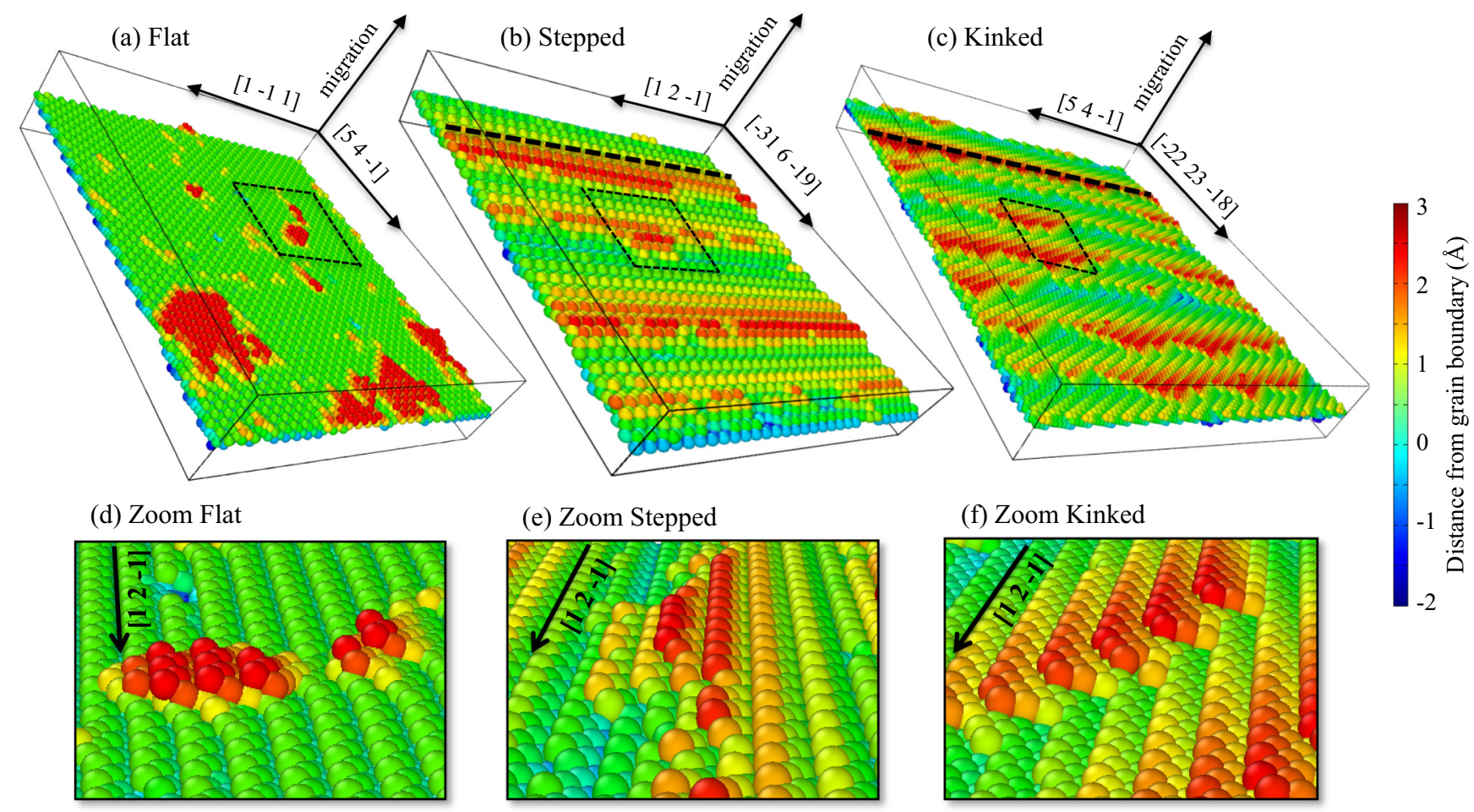

FIG. 5. (a), (b), and (c) are snapshots of migration in the three grain boundaries [(a) flat, (b) stepped, and (c) kinked] at $500 \mathrm{ps,} 3 \mathrm{meV}$, and $450 \mathrm{~K}$ where the bulk atoms have been removed and the atoms belonging to the shrinking grain have been made invisible to help resolve the atomistic details on the growing side of the boundaries. (d), (e), and (f) are the magnified views of the areas indicated in the snapshots. The black arrows indicate the $\left[\begin{array}{lll}1 & 2 & 1\end{array}\right]$ direction in all grain boundaries.

For the stepped boundary, step flow via double kink nucleation and propagation is the fundamental migration mechanism. It naturally explains the stop-and-go mode of this boundary shown in Fig. 2(a) and discussed in Sec. III B. The propagation of this grain boundary corresponds to a total of many such events on different steps explaining the smeared out behavior of the stop-and-go mode in Fig. 2(a).

One can intuitively understand that a double kink nucleation process has a lower energy barrier compared with the island nucleation on the flat boundary as suggested in Sec. III B. In fact, a careful analysis of the islands on the flat boundary [see Fig. 5(d)] reveals that an island nucleus can be perceived as an island of monoatomic double kinks nucleating on the $\left\{\begin{array}{lll}1 & 1 & 1\end{array}\right\}$ planes elongating in the [ $\left[\begin{array}{lll}1 & 2 & 1\end{array}\right]$ direction. These double kinks are thermodynamically unstable until they accumulate and form an island of a critical size, above which they can grow.

The fact that on the kinked boundary the originally [5 $4 \overline{1}]$ oriented steps subfacet into [ $\left[\begin{array}{lll}2 & \overline{1}\end{array}\right]$ and [ 110$]$ directions implies that these directions are low in energy and form cusps in the Wulff shape of possible steps on this boundary. The propagation of this boundary occurs via a kink flow mechanism through the geometrically necessary single kinks as opposed to the double kinks of the stepped boundary. In contrast to the other two boundaries, the flat and the stepped one, no nucleation mechanism is required.

This atomistic insight immediately explains the migration behavior shown and discussed in Sec. III B, i.e., a migration event with an activation barrier that is independent of temper- ature and driving force. We can quantitatively describe this mechanism using the harmonic-transition-state equation [39],

$$
v=v_{0} \exp \left(-E_{\text {barrier }} / k_{B} T\right) \text {. }
$$

Assuming a typical attempt frequency $v_{0}=10^{13} \mathrm{~s}^{-1}$ and temperature $T=500 \mathrm{~K}$, a single kink flow event occurs with a rate of $v \approx \mathrm{ps}^{-1}$. Multiplying this number with the number of available kink sites for the kinked boundary $(\approx 200)$, kink flow events are frequent on the simulation time scale of a few hundred ps. This explains why the overall position versus time dependence of the kinked boundary shows a smooth, continuous dependence [cf. Fig. 2(a)] even down to very low temperatures.

\section{DISCUSSION}

Table II summarizes the characteristic features of the migration behavior of the three representative grain boundaries as discussed in Sec. III. Additionally, the last column of Table II indicates the probability that these grain boundary structures exist in the vicinity of the $\Sigma 7$ symmetric tilt boundary. According to our simulation results the majority of mixed grain boundaries contain geometrically kinked steps and follow a qualitatively similar kinetics to the studied kinked boundary in this study. The stepped boundary consisting of straight steps is a rare case. Straight steps are only stable if the inclination axis lies along high symmetry directions such as $\langle 121\rangle$.

The structure of the kinked boundary closely resembles the terrace-ledge-kink model for a general high-angle grain 
TABLE II. Characteristic features of the three representative grain boundaries. "Asymptotic barrier" is the migration barrier height at the limit of zero driving forces. "Occurrence" indicates how frequently such a grain boundary is observed among the studied grain boundaries.

\begin{tabular}{lllccc}
\hline \hline Type & Structure & \multicolumn{1}{c}{ Atomistic feature } & Nucleation & Arrhenius & Asymptotic barrier \\
\hline Flat & symmetric tilt & islands of double kinks & yes & no & singular \\
Stepped & mixed $\langle 121\rangle$ & double kinks & yes & yes & $\approx 0.3 \mathrm{eV}$ \\
Kinked & mixed $\langle 541\rangle$ & single kinks & no & yes & $\approx 0.1 \mathrm{eV}$ \\
\hline \hline
\end{tabular}

boundary originally introduced by Gleiter [2,3]. In contrast to the original model of Gleiter, the explicit calculations show that emission and absorption of atoms does not occur through an intermediate layer but rather they occur simultaneously by the rearrangement of a few atoms at the kink sites. Gleiter's notion of the crystallography of the steps does not fully resemble our observation of nanofaceted structures, but his emphasis on the role of $\left\{\begin{array}{lll}1 & 1 & 1\end{array}\right\}$ planes in the migration of an fcc metal is corroborated by the results in Sec. III C.

The double kink nucleation process, as revealed here for the stepped boundary, has not been reported as a rate determining process in the motion of realistic grain boundaries so far. However, as mentioned in the introductory section it has been extensively discussed in the dynamics of two-dimensional extended defects: for example the motion of model grain boundaries and dislocations [10,11,40]. Double kink nucleation and propagation has also been repeatedly observed and reported in studies of surface growth [41]. A recent detailed 3D analysis of molecular dynamics simulations showed that crack propagation in brittle materials and at low temperatures occurs via double kink nucleation and subsequent kink propagation [42].

\section{A. Kinetic model for double kink nucleation on steps}

For a grain boundary migrating via step propagation, the kinetics is determined by the nucleation and subsequent growth of double kinks. To develop a kinetic model of this mechanism, let us first consider an idealized geometry of the formation of a double kink [Fig. 6(a)]. The green part schematically represents the grain boundary area before the formation of the double kink, while the double kink is indicated by the red area. For the following discussion we approximate the formation energy $E_{f}^{\mathrm{dk}}$ of a single double kink as

$$
E_{f}^{\mathrm{dk}}=E^{\mathrm{cost}}+E^{\mathrm{gain}},
$$

where $E^{\text {cost }}$ represents the energy increase due to the two newly formed surfaces each with an area $A$ as indicated in Fig. 6(a), and where $E^{\text {gain }}$ is the gain in energy due to transforming a portion of the crystal volume into the grain favored by the driving force. The latter term depends on the volume of the double kink. In the following we specifically use

$$
E^{\text {gain }}=p A d,
$$

where $p$ is the driving force and $d$ the length of the double kink. To derive an expression for $E^{\text {cost }}$ as a function of $d$ we first note that $E^{\operatorname{cost}}(d)$ should obey the following conditions

$$
E^{\text {cost }}(d)= \begin{cases}0 & \text { for } d=0 \\ \text { const. } & \text { for } d \rightarrow \infty,\end{cases}
$$

which reflect that at $d=0$ no double kink exists and that for large separations the interaction energy between the two kinks vanishes. Next we derive a functional dependence between these two limits. A simple $-1 / d$ term does not satisfy the $d=0$ limit and introduces an unphysical, singular behavior. To satisfy these conditions we use a Fermi-Dirac-like function. From a number of possible functions, Fermi Dirac was chosen because it has the correct asymptotic limits and its smoothness can be easily controlled:

$$
E^{\text {cost }}(d)=E_{\infty}\left(\frac{-2}{1+\exp (a d)}+1\right) .
$$

Here, $E_{\infty}$ is the constant energy cost to which Eq. (7) converges for $d \rightarrow \infty$ and $a$ is a parameter that determines the smoothness of the Fermi-Dirac function. The black dashed line in Fig. 6(b) shows an example for the dependence of $E^{\text {cost }}(d)$. Using Eqs. (5) and (7) in Eq. (4) we obtain

$$
E_{f}^{\mathrm{dk}}(d, p)=E_{\infty}\left(\frac{-2}{1+\exp (a d)}+1\right)+p d A .
$$

The solid lines in Fig. 6(b) show examples of the dependence of $E_{f}^{\mathrm{dk}}(d)$ for three different driving forces $p$. Similar to a classical 3d-nucleation model there exists a critical distance
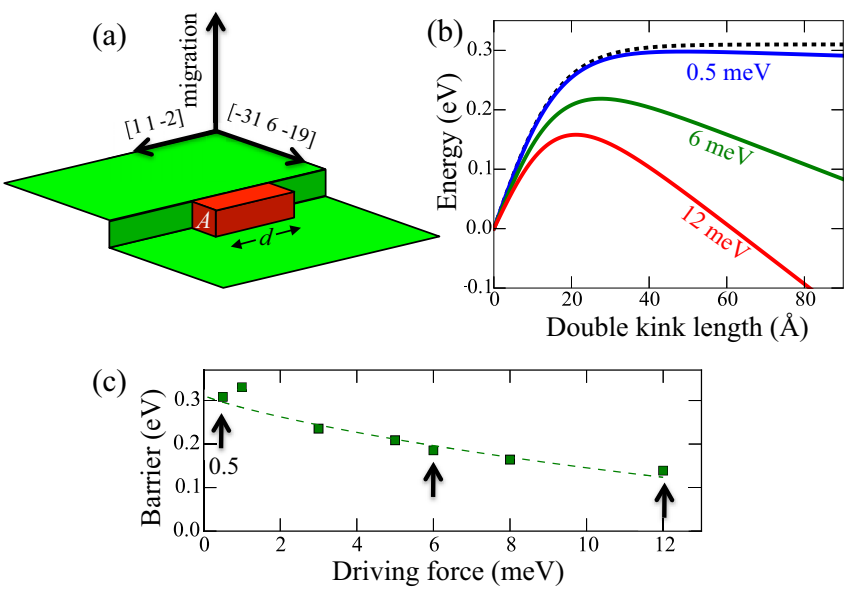

FIG. 6. Geometry and energy barrier of the double kink nucleation process. (a) A double kink of length $d$ on a stepped grain boundary and with a kink area $A$. (b) The formation free energy of the double kink as a function of kink separation as derived with the nucleation model [Eq. (8)] at different driving forces. The black dotted line shows the dependence in the absence of any driving force. (c) Compares the activation barrier as derived from the MD simulations at different driving forces (filled green squares) and the analytic nucleation model (green dashed line). The arrows mark the driving forces for which the explicit energy curves are shown in (b). 
$d^{\text {crit }}$, above which the double kinks become thermodynamically stable and below which they are subcritical, i.e., they are likely to annihilate. The resulting maximum of each curve corresponds to the nucleation barrier at the given driving force as indicated by the three arrows in Fig. 6(c).

To be able to use the developed model for fitting the dependence of the nucleation barrier as a function of the driving force, Eq. (8) needs to be transformed using an explicit critical length. The latter is determined from $\partial E_{f}^{\mathrm{dk}} / \partial d=0$ giving

$$
d^{\text {crit }}=\frac{f(x, p)}{a}, \quad f(x, p)=\operatorname{arcCosh}\left(\frac{x}{p}-1\right),
$$

with $x=a E_{\infty} / A$. Using $d^{\text {crit }}$ in Eq. (8) gives

$$
E_{f}^{\mathrm{dk}}(p)=E_{\infty}\left(\frac{-2}{1+\exp [f(x, p)]}-\frac{p}{x} f(x, p)\right) .
$$

An important point is that only two free parameters, the kink formation energy $E_{\infty}$ and the ratio $x$ defined above, are needed to obtain the activation barrier $E_{f}^{\mathrm{dk}}(p)$ as a function of driving force $p$. Applying the kink nucleation model [Eq. (10)] to our computed migration data (see Fig. 4) we find that it accurately fits the driving force dependence of the barriers for the stepped boundary [Fig. 6(c)].

Using the fit shown in Fig. 6(c) we directly obtain the parameters for the nucleation model. Specifically, we get the asymptotic limit for the double kink formation $E_{\infty}=0.31 \mathrm{eV}$ and the ratio $x=0.005 \mathrm{eV} / \AA^{2}$. The fact that the asymptotic value of the migration barrier at zero driving force is identical to the double kink formation energy $E_{\infty}$ can be intuitively understood - with decreasing the driving force the kink separation distance increases to infinity and the nucleation barrier approaches $E_{\infty}$ [see Fig. 6(b)].

Having the ratio $x$ allows us to determine the other parameters entering the model. We obtain the critical double kink size $d^{\text {crit }}$ from the MD simulations of the stepped surface and use Eq. (9) to derive the kink-kink interaction parameter $a$. To determine the critical size we analyzed the probability distribution of the double kink length in the migration snapshots. This distribution sharply increases from low probability (which is expected for subcritical double kinks) to high probability (thermodynamically stable double kinks) when the double kink separation goes above the critical length. At a driving force of $6 \mathrm{meV} /$ atom we find a critical value of $d^{\text {crit }} \approx 20 \AA$ which corresponds to a double kink consisting of $\approx 4$ atoms. Using Eq. (9) and the fitted value for $x$ results in $a=0.15 \AA^{-1}$ and $A=9.3 \AA^{2}$. As a cross-check, the value of $A$ can be compared to a square with side lengths of the nearest neighbor distance in fcc resulting in an area of $\approx 8 \AA^{2}$.

\section{B. Parallels to surface growth}

Many of the structural and dynamical aspects that have been identified and discussed in this study have a close

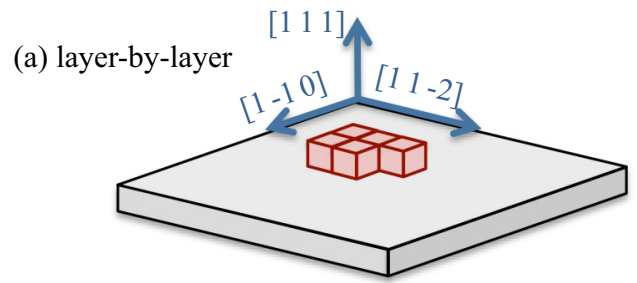

(b) vicinal-straight step

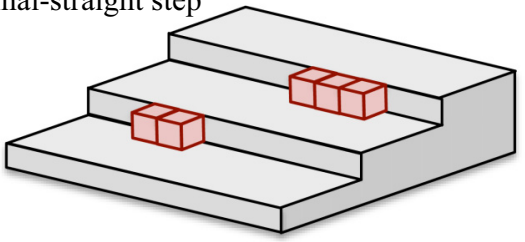

(c) vicinal-general

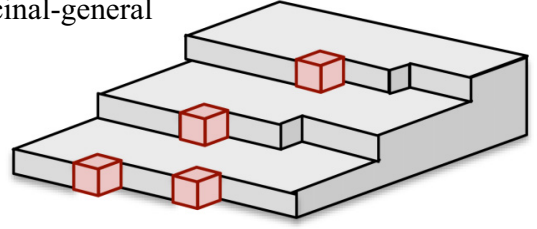

FIG. 7. (a), (b), and (c) are surface growth parallels to the grain boundaries of Figs. 5(a), 5(b), and 5(c), respectively. (a) represents a layer-by-layer growth and (b) and (c) are vicinal surfaces that grow via a step flow mechanism.

resemblance to concepts well known in surface growth. In fact, this similarity largely inspired our study. In the past this close connection between grain boundary migration and the growth of crystalline surfaces was rarely utilized. However, to understand the importance of the mesoscale structure/surface morphology on growth rates of grain boundaries it is helpful to connect to the vast knowledge that has been accumulated in surface science. In the following we will therefore provide a brief discussion about similarities, but also differences, between the concepts in these two fields.

Island nucleation [Fig. 7(a)] is observed in surface growth when deposition happens on flat close-packed surfaces [e.g., ( $\left.\begin{array}{lll}1 & 1 & 1\end{array}\right)$ or $\left(\begin{array}{lll}1 & 0 & 0\end{array}\right)$ in fcc] with no step character (zero or low miscut angles). In the terminology of surface epitaxy this growth mode is referred to as layer by layer growth. When the surface is vicinal to a low energy cusp orientation such as (111), it may contain straight (faceted) steps along high symmetry directions and grow via double kink nucleation and propagation as indicated in Fig. 7(b). A more general vicinal surface can contain geometrically necessary kink sites and grow via the flow of kinks on the steps as shown in Fig. 7(c). The growth modes via double kink nucleation and propagation and the flow of kinks [Figs. 7(b) and 7(c)] are referred to as step flow in surface epitaxy. These types of vicinal surfaces have been observed using scanning tunneling microscopy under equilibrium conditions [43] or during growth [44].

From a structural point of view and based on our observations in this study we can conclude that the flat grain boundary equivalent of Fig. 7(a) is the symmetric tilt grain boundary plane, in other words the plane of mirror symmetry between the two crystals [30]. The grain boundary equivalent of Fig. 7(b) is rarely observed and occurs only when a high symmetry direction such as $\langle 121\rangle$ exists between the two crystals and 
finally the equivalent of Fig. 7(c) is a grain boundary with steps along a low symmetry direction in the bicrystal. The latter case constitutes the majority of the grain boundary geometries inspected in this study.

Despite the apparent geometric parallels, there are also important qualitative differences between surface epitaxy and grain boundary migration. Surface growth can be deemed as a process farther from equilibrium and therefore long range diffusion on surfaces plays an important role in determining the growth modes. The growth modes can change regardless of the original surface geometry whereas in grain boundary migration a long range diffusion process is nonexistent and atomic shuffling near the boundary is responsible for the motion.

\section{Comparison with experiment}

Having an explicit analytical kinetic model for all three considered boundary types, i.e., flat, stepped, and kinked, enables us to determine the migration barriers at the experimentally relevant conditions (driving forces $<1 \mathrm{MPa}=0.1 \mathrm{meV} /$ atom). Experimental studies of shear coupled motion on $\Sigma 7$ bicrystals in $\mathrm{Al}$ [25] resulted in activation energies in the range of 0.67 to $1.3 \mathrm{eV}$ [black bar in Fig. 4(a)].

For the flat boundary the migration barriers are inversely proportional to the driving forces as discussed in Sec. III B and shown in Fig. 4(a) (red dashed line), i.e., they go to infinity at vanishing driving forces. An extrapolation down to $0.1 \mathrm{meV} /$ atom yields a barrier of $90 \mathrm{eV}$. Having such a barrier corresponds to one nucleation event per $10^{1000}$ years [using Eq. (3) with $\nu_{0}=10^{13} 1 / \mathrm{s}$ and $T=500 \mathrm{~K}$ ]. Thus, at experimental driving forces and below the roughening temperature (above it the boundary would no longer be flat but spontaneously form steps and kinks) a perfect flat boundary would be completely immobile. The fact that this boundary is found to migrate in experiments [25] indicates that the grain boundary in the bicrystal is not perfectly symmetric down to the atomistic scale but rather it contains structural defects such as kinks or steps.

As derived in this study, for the stepped and kinked boundaries the barriers at the experimental driving force limit are $\approx 0.3 \mathrm{eV}$ and $\approx 0.1 \mathrm{eV}$, respectively; Fig. 4 . The former limit is obtained from our double kink nucleation fit and the latter from a constant linear fit (as the barrier does not depend on the driving force). These barriers are substantially lower than the experimental values, about a factor of 2 to 3 for the stepped boundary and an order of magnitude for the kinked boundary. Such large deviations are unlikely to be resolved by using a better theoretical description (i.e., ab initio calculations). However the impurity drag, as often speculated [45], can contribute to this discrepancy. Even though the chemical purity of experimental Al samples in Ref. [25] is close to $99.9995 \%$, segregation of impurity atoms to the boundary is not negligible. They can pin the kink sites and alter the mechanisms and barriers. A future avenue for this study is to investigate this pinning effect in the motion of the kinked and stepped grain boundaries.

Another possible explanation for the discrepancy has roots in the theory of crystal growth: The kinked boundaries with lower migration barriers grow fastest and thus are bound to disappear first whereas the ones with high barriers will prevail. A realistic macroscopic grain boundary plane contains multiple plane inclinations and hence various step orientations. The regions that contain a high kink density are expected to quickly grow out of kinks. After some time the equilibrium boundary will mainly consist of the steps with low kink densities that require double kink nucleation with the highest barriers to progress. The above picture provides an additional source why the barriers for our fast moving kinked and stepped boundaries are below the experimental data. Thus a more systematic analysis of all relevant step configurations will be an important topic for future studies.

\section{CONCLUSIONS}

We have investigated the migration kinetics of mixed tilt and twist grain boundaries in the vicinity of a symmetric $\Sigma 7$ tilt orientation. Performing a careful analysis we selected three representative types of boundaries that, when relaxed, form nanofacets/steps along different orientations. Steps along a high symmetry direction in the bicrystal such as the $\left\langle\begin{array}{lll}1 & 21\rangle \text { will }\end{array}\right.$ be straight whereas the ones along low symmetry directions such as, e.g., $\langle 541\rangle$ form geometrically necessary kinks. The symmetric tilt grain boundary will have a step-free atomicallyflat structure.

We studied the migration behavior of these three boundary types-atomically flat, stepped, and kinked-using sizeconverged molecular dynamics simulations. Performing these calculations for a wide range of driving-forces and temperatures, we derived the dependence of the migration barrier as a function of driving force. Our results show qualitatively different relations for the three grain boundaries. The respective relations have been explained as a direct consequence of the rate limiting atomic migration mechanisms: The nucleation of islands or double kinks for the flat and stepped boundaries, respectively, and propagation of the already existing kinks for the kinked boundary. The absence of a nucleation mechanism for the kinked boundary naturally explains its driving forceindependent migration barrier.

With the knowledge of the identified atomistic mechanisms, we were able to analytically describe the driving force dependence for the three representative boundaries. From the analytic relations we obtained the asymptotic limits of the migration barriers at zero driving force. While this value is infinity for the flat boundary, we find finite values for the two other interfaces, $\approx 0.3 \mathrm{eV}$ for the stepped boundary and $\approx 0.1 \mathrm{eV}$ for the kinked one. This knowledge enabled us to discuss the migration barriers of grain boundaries at small experimental driving forces without running prohibitively long molecular dynamics simulations.

We have learned that inspecting the static mesoscale structure of a specific boundary will be sufficient to identify its structure, i.e., stepped or kinked, and therefore to predict and model their respective migration behavior.

The approach introduced here to derive the asymptotic behavior of the migration barrier for vanishing driving forces based on the identification of the rate limiting atomistic mechanism is general and can be applied to arbitrary grain boundaries. 


\section{ACKNOWLEDGMENTS}

We would like to thank L. Lymperakis and A. Stukowski for fruitful discussions. R. Hadian was funded by an Alexander Von Humboldt post doctoral fellowship. This project has also received funding from the European Research Council (ERC) under the European Union's Horizon 2020 research and innovation programme (Grant No. 639211).
[1] N. F. Mott, Proc. Phys. Soc. 60, 391 (1948).

[2] H. Gleiter, Acta Metall. 17, 565 (1969).

[3] H. Gleiter, Acta Metall. 17, 853 (1969).

[4] G. Gottstein and L. Shvindlerman, Grain Boundary Migration in Metals: Thermodynamics, Kinetics and Applications, 2nd ed. (CRC Press, Boca Raton, FL, 2010).

[5] D. A. Smith and R. C. Pond, Intl. Metals Rev. 21, 61 (1976).

[6] R. C. Pond, Proc. R. Soc. London A 357, 471 (1977).

[7] C. M. F. Rae and D. A. Smith, Philos. Mag. A 41, 477 (2006).

[8] S. E. Babcock and R. W. Balluffi, Acta Metall. 37, 2367 (1989).

[9] M. I. Mendelev and D. J. Srolovitz, Acta Mater. 48, 3711 (2000).

[10] M. I. Mendelev, D. J. Srolovitz, L. S. Shvindlerman, and G. Gottstein, J. Mater. Res. 17, 234 (2011).

[11] A. E. Lobkovsky, A. Karma, M. I. Mendelev, M. Haataja, and D. J. Srolovitz, Acta Mater. 52, 285 (2004).

[12] H. Zhang and D. J. Srolovitz, Acta Mater. 54, 623 (2006).

[13] H. Zhang, D. J. Srolovitz, J. F. Douglas, and J. A. Warren, Acta Mater. 55, 4527 (2007).

[14] C. P. Race, J. von Pezold, and J. Neugebauer, Phys. Rev. B 89, 214110 (2014).

[15] J.-K. Kim, Adv. Mater. 5, 685 (1993).

[16] C.-S. Kim, A. D. Rollett, and G. S. Rohrer, Scr. Mater. 54, 1005 (2006).

[17] D. M. Saylor, B. S. El Dasher, A. D. Rollett, and G. S. Rohrer, Acta Mater. 52, 3649 (2004).

[18] D. L. Olmsted, E. A. Holm, and S. M. Foiles, Acta Mater. 57, 3704 (2009).

[19] E. R. Homer, S. Patala, and J. L. Priedeman, Sci. Rep. 5, 15476 (2015).

[20] E. R. Homer, E. A. Holm, S. M. Foiles, and D. L. Olmsted, JOM 66, 114 (2013).

[21] J. A. Brown and Y. Mishin, Phys. Rev. B 76, 134118 (2007).

[22] M. A. Tschopp and D. L. McDowell, Philos. Mag. 87, 3871 (2007).

[23] I. Toda-Caraballo, P. D. Bristowe, and C. Capdevila, Acta Mater. 60, 1116 (2012).
[24] C. P. Race, R. Hadian, J. von Pezold, B. Grabowski, and J. Neugebauer, Phys. Rev. B 92, 174115 (2015).

[25] D. A. Molodov, T. Gorkaya, and G. Gottstein, Scr. Mater. 65, 990 (2011).

[26] R. R. Zope and Y. Mishin, Phys. Rev. B 68, 024102 (2003).

[27] D. A. Molodov, U. Czubayko, G. Gottstein, and L. S. Shvindlerman, Acta Mater. 46, 553 (1998).

[28] K. G. Janssens, D. Olmsted, E. A. Holm, S. M. Foiles, S. J. Plimpton, and P. M. Derlet, Nat. Mater. 5, 124 (2006).

[29] S. Plimpton, J. Comput. Phys. 117, 1 (1995).

[30] A. Sutton and R. Balluffi, Interfaces in Crystalline Materials (Clarendon Press, Oxford, 1996).

[31] D. L. Olmsted, S. M. Foiles, and E. A. Holm, Scr. Mater. 57, 1161 (2007).

[32] S. Noseé, J. Chem. Phys. 81, 511 (1984).

[33] W. G. Hoover, Phys. Rev. A 31, 1695 (1985).

[34] M. Parrinello, J. Appl. Phys. 52, 7182 (1981).

[35] A. Stukowski, Modell. Simul. Mater. Sci. Eng. 18, 015012 (2010).

[36] A. Stukowski, V. V. Bulatov, and A. Arsenlis, Modell. Simul. Mater. Sci. Eng. 20, 085007 (2012).

[37] Z. X. Wu, Y. W. Zhang, and D. J. Srolovitz, Acta Mater. 57, 4278 (2009).

[38] J. W. Cahn, Y. Mishin, and A. Suzuki, Acta Mater. 54, 4953 (2006).

[39] G. Henkelman, G. Jóhannesson, and H. Jónsson, in Progress on Theoretical Chemistry and Physics (Kluwer, Dordrecht, 2000), pp. 269-300.

[40] J. P. Hirth and J. Lothe, Theory of Dislocations (Wiley Interscience, New York, 1982).

[41] Handbook of Crystal Growth, Fundamentals: Thermodynamics and Kinetics, 2nd ed. (Elsevier, Amsterdam, 2015).

[42] F. Rösch and H. R. Trebin, Europhys. Lett. 87, 66004 (2009).

[43] M. Giesen, Prog. Surf. Sci. 68, 1 (2001).

[44] M. H. Xie, S. M. Seutter, W. K. Zhu, L. X. Zheng, H. Wu, and S. Y. Tong, Phys. Rev. Lett. 82, 2749 (1999).

[45] M. I. Mendelev, C. Deng, C. A. Schuh, and D. J. Srolovitz, Modell. Simul. Mater. Sci. Eng. 21, 045017 (2013). 